\title{
Effect of Biomaterial Prosthetics on the Rehabilitation of Lower Limb Amputees
}

\author{
Qaysar Mohi Ud Din* \\ Department of Biomedical Engineering, Bharath University, India
}

Received: September 08, 2017; Published: September 18, 2017

*Corresponding author: Qaysar Mohi Ud Din, Department of Biomedical Engineering, Bharath University, Chennai-500073, Tamil Nadu, India; Email: qaysar007@yahoo.in

\begin{abstract}
The prosthetics is done in order to help the persons with various degrees of amputation to regain their normal functioning. The prosthetics used for rehabilitation (regaining of body functionality) should be comfortable for the patient and should be durable. After lower limb prosthesis, the most important goal in the rehabilitation process of the amputee is the regaining of walking. The walking ability is very essential for proper functioning. There are two different types of foot available that are used based on function. These are the dynamic and non dynamic response foot (e.g. solid ankle cushion heel). The non dynamic foot is basic for appearance and the functions such as standing and walking, while the dynamic can be used for more intense activities like running. Current prosthetic foot designs do not exactly match the characteristics of normal human foot. Human foot is a multi-functional device that performs various activities, but the functions of prosthetic feet are limited to only few. A person's prosthesis should be designed and assembled according to the patient's appearance and functional needs. Over the years there have been significant advancements in prosthetics. New plastics and other materials, such as carbon fiber, have made artificial limbs stronger and lighter, reducing the amount of extra energy necessary to operate the limb. This is very important for trans-femoral amputees. Additional materials have made artificial limbs to look much like real limbs, which is important to trans-radial and trans-humeral amputees because they are more likely to have the artificial limb exposed. The foot provides shock absorption and stability during stance by providing contact to the ground. Additionally it influences the gait biomechanics by its shape and stiffness. The basis of this project is to provide standing and walking functions as well as to make the appearance attractive. The available below knee prosthesis device employs silicon material for the foot which is imported and expensive; hence there is a need to use a cost effective and easily available material (wood). The main problem associated with current feet is durability and endurance. The different characteristics on the basis of which the prosthetics are used include: cosmetics, cost, and ease of use, size availability, weight, rotation, and energy absorption and ground compliance. The objective of this project is to design a prosthetic feet model that can function as a normal human foot, whose characteristics match the most with the normal human foot and to evaluate the functioning of prosthetics in rehabilitation. The designed prosthetic feet model can then be used to evaluate the durability, endurance and comfort level provided by the designed SACH prosthetic foot. On the basis of this model further studies can be conducted to evaluate the effect of prosthetic feet in the rehabilitation of patients with lower limb prosthesis.
\end{abstract}

Keywords: Prosthetics; Amputee; Gait; Trans-Humeral; Rehabilitation

Abbreviations: FEA: Finite Element Analysis; PVA: Polyvinyl Acetate Rubber

\section{Introduction}

Prosthesis is an artificial device that replaces a missing body part, which may be lost through trauma, disease, or other congenital conditions. Prosthetic amputee rehabilitation is done by trained professionals like prosthitist and an inter-disciplinary team of health care professionals including physiotherapists. Current prosthetic foot designs do not exactly replicate the characteristics of a normal human foot. A human foot is a multi-functional unit that can perform a wide range of activities. However, a prosthetic foot is limited to only a few activities. More recently, manufacturers of prosthetic feet have looked into the characteristics of a prosthesis that may be adjustable. The amputee may then be able to perform a number of activities without requiring a different prosthesis. It is important to establish the characteristics of a human foot used in its functional operations. This investigation has limited the activities to normal gait cycle in walking, the most common use of a prosthetic foot [1-5].

The characteristics of a human and prosthetic foot covered in the scope of this investigation are dorsiflexion, eversion, impact absorption and the torque generated at the ankle. These are the most important characteristics in determining an appropriate prosthesis, according to requirement of an amputee. This investigation is aimed at designing a prosthetic foot that incorporates prosthetic 
design elements currently available, in order to design and develop a new prosthesis. The major reasons that cause an amputation of the lower limbs are: Disease, Trauma, Congenital or Birth defects, and Tumors. Specifically, the conditions that lead to amputation of an extremity of the body can be:

i. impaired circulation as a complication of diabetes mellitus

ii. hardening of the arteries

iii. arterial embolism

iv. gangrene

v. severe frostbite

\section{Prosthetic Feet}

Prosthetic feet are very important for regaining the walking ability by the persons who have lost this ability due to some injury or by some other causes. Each foot serves to provide for the needs of a slightly different user than the next. A prosthetic provides a stable weight-bearing surface, absorbs shock, replaces lost muscle function, replicates the anatomic joint, and restores cosmetic appearance ('Lower Limb Prosthetics'). Users of prosthetic feet can be broken down into functional levels which relate to their activity level and needs for their prostheses. A Level 0 amputee does not have the ability to go through the gait cycle due to massive injuries. A prosthetic would not help someone at a Level 0. A Level 1 has the ability to ambulate, but does not have the stability to walk on sloped ground. A Level 2 amputee can travel on some varying terrain and slopes. A Level 3 can traverse ranging slopes and conditions. They have varying activity levels. The last level, a Level 4, has high mobility and will subject their prosthetic to high impact and high stress situations [6-9].

\section{Prosthetic Foot Characteristics}

One of the key factors in designing a new prosthesis is the analysis of an amputee's response towards the prosthesis and the ease with which the prosthesis can be used. The characteristics which are important in achieving natural gait motion include:
A. Energy Return
B. Dorsiflexion
C. Ankle Torsion
D. Eversion
E. Impact Absorption

\section{Component And Characteristics Of Prosthetic Feet}

Characteristics of prosthetic feet are not only represented by its material properties but also by the component and orientation of such components. A prosthetic foot consists of a heel, keel, ankle adaptor and a cosmoses. These components vary in geometry, orientation and material composition in each prosthesis and are to be according to their specific function [8-14]. The characteristic analysis of individual prostheses is based on the desired characteristics inherent during normal walking. The components of the prostheses are examined in order to analyze the origin of the generated characteristic.

\section{Keel}

The function of a keel within prosthesis is to provide the energy transfer from the heel strike through to the toe off and the dorsiflexion required for natural ambulation. Depending upon the quantity of surrounding foam it also provides rotational properties such as eversion and torsion.

The keel to be used in the new prosthesis is of a modified SACH Foot design using a Delran II, nylon composite material, and intermediate polyurethane rubbers. Testing indicates that the $\mathrm{SACH}$ foot keel design generates the desired characteristics more favorably than other prosthetic feet. A narrow ankle block of SACH IS51, the keel will be designed in order to provide for a larger torque [15].

Through heel impact testing, the adaptation of a section to the keel improves the impact absorption characteristics of the prosthesis. Therefore to design the section into the keel it would also increase the impact absorption of the new prosthesis.

Heel

a. The function of a heel within prosthesis is to provide the impact absorption at heel strike and also provides the kinetic energy required for a smooth transition between the heel strike and the toe off.

b. The heel to be used in the new prosthesis is SACH heel wedge which utilizes a low density, sponge like, polyurethane. Through impact testing, SACH wedge indicated the greatest energy storing potential which is used to increase the amount of dorsiflexion produced by prosthesis.

c. The triangular wedge itself provides for greater absorption due to the larger end being exposed to the heel strike. Used in conjunction with the section, the combined components provide extra impact absorption

d. The function of the filler is to provide durable and aesthetically pleasing cosmoses also well as complementing the other component in performing the desired characteristics. The SACH Feet uses a high grade, medium density polyurethane foam.

e. The filler density and quantity is important in torsion about the ankle as the keel requires the ability to twist. The foam density and quantity either side of the keel provides the eversion properties of prosthesis.

f. Excessive filler foam may alter the performance characteristics of the individual components. As a result the filler above the keel must be kept to a minimum in order to retain the elastic properties of the keel.

\section{Experimental Methods \\ Materials}

The following materials were chosen to make the wooden foot below knee prosthesis help in rehabilitation, light in weight, durable, low cost and to provide shock absorption. The materials chosen for the fabrication of the wooden foot are easily available. 
Wood: It has even grain pattern, few voids, strength, durability and light weight and can be given any desired shape.

Glue: It is used to join the rubber toe to rest of the foot, forming the metatarsal joint.

Leather: It is used to protect the wooden foot from soaking and it is easy to clean.

Sole rubber: It is a thicker rubber used to make the sole of the foot.

Rubber: It is used to make the cushion heel of the foot.

Acrylic paint: It used to paint the rubber toe to fit the color of the leather.

Fiber glass: It is used to make the socket stronger.

Plaster Of Paris bandage: It is used to obtain the outline of the stump.

Plaster Of Paris powder: It used to form the positive model of the stump.

Polyvinyl acetate rubber (PVA): It is used to laminate the socket.

Cloth: It carries the picture of the cartoon character for the socket Water, to dissolve the Plaster of Paris when forming the positive mold.

Polyester resin: It forms the laminated plastic socket when it hardens.

\section{Methodology}

The patients with lower limb amputations are made to feel welcome and comfortable. The patient with amputations are analyzed to get all the necessary details from them. Before doing any prosthesis there should the required data available about the level of amputation. The measurements of the amputated part should be available for the designing and the application of the prosthesis. The tracing and measurement is inverted on the wood to get the shape and size needed for the making of the foot. Wood is acquired for the foot fabrication. The wood is lighter in weight compared to the silicone material, it is also durable and easy to work with.

A. The wood is carved to the shape and size obtained from the measurement of the patient's left foot.

B. The toe is shaped to look like a real toe, with glue the toe is fitted to the foot at the metatarsal joint.

C. The heel part of the wooden foot is carved out from SACH rubber, with glue the toe is fitted to the carved wood, the cushion heel is made for the purpose of shock absorption on heel contact.

D. A negative mold of the stump is made by wrapping it with a wet plaster of Paris bandage; it is rubbed around the stump with the arm to make sure there is no space between the Plaster Of Paris bandage and the stump.

E. A positive model of the stump is made by filling the cast with a mixture of plaster of Paris powder and water.
F. After the cast has solidified, modifications are made to the model to make sure that the pressures points are marked correctly.

G. The fiber glass, cloth and PVA rubber are wrapped around the positive model then a mixture of plastic resin and hardener is poured between the PVA rubber and cloth.

H. After the plastic resin hardens, a laminated picture of the cartoon characters is obtained.

I. The adjustable pylon is fitted into the foot at one end and at the other end it is attached to the socket by means of screws. The pylon has a nut and screw for the adjustment in different holes. The stability of the prosthesis is checked using the bench alignment before conducting walking trials.

\section{ISO Standards}

There are two standards that outline procedures for testing prosthetic leg and foot systems. These ISO standards are created for prosthetics to ensure that prostheses are adequate and safe for users. The first is ISO 10328 [Structural testing of lower-limb prostheses]. According to ISO 10328, 'the term prosthetic means an externally applied device used to replace wholly, or in part, an absent or deficient limb segment.' The standards specify testing methods, loading conditions, and other parameters. The various standards in ISO 10328 apply to trans-tibial knee-disarticulation, and trans-femoral knee prostheses (ISO 10328 2006). There are three types of testing structures indicated in ISO 10328: complete, partial, and any other structure. This research primarily dealt with the complete structure testing. In preparation for testing, all cosmetic components must be removed if they do not provide structural strength to the device (ISO 10328 1996).

The products being tested are fitted normally, further description on this method can be found in the methodology. The 2006 ISO 22675 [Testing of ankle-foot devices and foot units] standards is used more heavily in the research for this project. This ISO standard discusses the proper procedure for cyclic testing of ankle-foot devices. It reviews the loading conditions that can be used to mimic natural gait loading. In addition to the cyclic loading tests, the ISO 22675 specifies a static test that can be performed on prosthetic ankle-foot devices. It shows appropriate lines of action for the static loading tests (ISO 22675 2006). During testing the sample must be aligned appropriately being aware of the effective ankle joint centerline, the effective ankle joint center, the effective knee joint centerline, and the effective knee joint center. All tests should be conducted using the standard's outlined worst case alignment. Through the use of ISO standards and following proper protocol, one can have a better chance of obtaining valuable results for a wide range of applications. The standards can also ensure safety during testing.

\section{Results}

From the physical testing and FEA models, data are collected regarding the strain on the mono-limb and the compression of the prosthetic assembly. In addition, information collected from the FEA included the principal stresses on the mono-limb and the von 
Misses stresses. Evaluation of the foot prosthetics is conducted in two partially concurrent phases in order to determine and compare the stiffness of the prosthetic systems and the stresses that occur on the foot. The first phase is analytical modeling the foot/monolimb assemblies and conducting finite element analysis (FEA). The second phase is the validation.

\section{Physical Testing}

The physical testing that is performed is directed at establishing the reactions that the mono-limb displayed when under the specified loading conditions. Each foot and mono limb combination is loaded as indicated in the methodology. The uni axial gauge located on the posterior seam of the mono-limb, and three strain gauges of the rosette are read and recorded.

\section{Heel-Strike}

During the heel-strike test for the assembly with the SR foot is stopped at a load of $910 \mathrm{~N}$ because plastic deformation began to occur near the heel of the foot. This deformation is shown as a bright white spot. The foot did not undergo fracture. The SACH foot is applied to the full $1300 \mathrm{~N}$ loading of the FEA through the physical testing of the feet.

\section{Determination of Axes For Prosthetic Components}

In order to ensure consistent and comparable measurements and results, it is important to determine the alignment for all of the testing components. The coordinates that are used to describe the alignment are the Cartesian coordinate system with positive $\mathrm{Y}$ in the upwards vertical direction, positive $\mathrm{X}$ in the right horizontal direction, and $\mathrm{Z}$ outwards.

\section{Discussion}

The analysis of the results shows initial heel strike compared to other trials is not good enough. We added more cushion to achieve the desired results. The outcome achieved is relatively very good as compared to the gait pattern of a normal human foot and the silicone foot which is expensive and is not readily available for every amputee due to high cost and maintenance. The gait analysis of the different phases (heel strike, total floor contact, mid stance, heel off and toe off, mid swing), and the metatarsal joint made toe off are possible. The total floor contact phase can be measured by using the bench alignment method and as the patient wore the below knee prosthetics, we obtain similar results and swing phase and stance phases are observed to be very good. We are able to achieve the designing and making of a cost effective below knee prosthesis by using materials that are easily available, like wood for the foot and an adjustable aluminum pylon.

\section{Conclusion}

A low cost below knee prosthesis for lower limb amputees has been fabricated. The silicon foot is substituted with the fabricated wooden foot which is covered with leather and acrylic paint, and artificial toe nails are fixed on the nail beds of the toe nails. The proper selection of the prosthetics for the rehabilitation of the amputees is very important. The prosthetic feet model which is created is cheap but limits the amount of functions or the movement of feet. There is no proper model of the prosthetics which are used but instead it depends on various factors. It can be concluded that if proper selection of heel stiffness and alignment of the prosthesis is done then, this type of prosthetic feet can be made to function so that similar whole body kinetic patterns can be obtained and it provides an advantage over the other prosthetic feet models as it be used by the patients who cannot afford other prosthetic designs and it is very easy to maintain. If costs and maintenance requirements are included as important factors in prosthetic foot selection, then the wooden SACH foot will be the first choice for the unilateral active below and above-knee amputees.

\section{Future Scope}

The available prosthetic foot model is used on the basis of various factors and there is still need for the designing of prosthetics which are more comfortable and which can resemble more closely with the natural foot. The current designs provide a great deal of movement of the foot and better prosthetic foot models need to be designed for the rehabilitation amputees. The different materials can be tested for making the prosthetics and prosthetic models which are more closely related to the functioning of the natural foot should be designed.

\section{References}

1. Alaranta H, Kinnunen A, Karkkainen M, Pohjalainen T, Heliovaara M (1991) Practical Benefits Of Flex-Foot In Below knee Amputees. J Prosthet Orthot 3: 179-181.

2. Arya AP, Lees A, Nirula HC, Klenerman LA (1995) Biomechanical Comparison Of The Sach, Seattle And Jaipur Feet Using Ground Reaction Forces. Prosthet Orthot Int 19(1): 37-45.

3. Barth, Daryl G, Schumacher, Laura BS, Thomas, et al. (1992) Gait Analysis And Energy Cost Of Below-Knee Amputees Wearing Six Different Prosthetic Feet. Journal of Prosthetics and Orthotics 4(2): 63-75.

4. Blumentritt S, Schmalz T, Jarasch R (2001) Influence of Static Prosthetic Alignment on Standing Posture and Walking In Transtibial Amputees. Orthopade 30(3): 161-168.

5. Doane, Street Gm, Caspers C (2001) A Comparison of Transtibial Amputee Suction and Vacuum Socket Conditions. Prosthet Orthot Int 25(3): 202-209.

6. Cortes A, Viosca E, Hoyos Jv, Prat J, Sanchez Lacuesta J (1997) Optimization of The Prescription For Trans-Tibial (Tt) amputees. Prosthet Orthot Int 21(3): 168-174.

7. Czerniecki JM, Munro CF, GitterA (1987) A comparison of the power generation/absorption characteristics of prosthetic feet during running. Arch Phys Med Rehabil 68: 636.

8. Charles M, Rasmussen J, Christensen ST, Surma E, de Zee M (2006) Analysis of musculoskeletal systems in the Any Body Modeling System. Simulation Modelling Practice and Theory 14(8): 1100-1111.

9. Edwards, Inman VT, BreslerB (1954) The principal elements in human locomotion. Human limbs and their substitutes, Hafner Publishing Co, New York, pp. 437-471.

10. Foort J (1965) The patellar-tendon-bearing prosthesis for below knee amputees : a review of technique and criteria. Artificial Limbs 13(1): 4-13.

11. Gordon EJ, Ardizzone J (1960) Clinical experiences with the S.A.C.H. foot prosthesis. Journal of Bone and Joint Surgery 42: 226-234.

12. Giakas G, Baltzopoulos V (1997) A comparison of automatic filtering techniques applied to biomechanical walking data. J. Biomechanics, 30(8): 847-850. 
13. Geil, Mark D (2001) Energy Loss and Stiffness Properties of Dynamic Elastic Response Prosthetic Feet. Journal of Prosthetics and Orthotics 13(3): 70-73.

14. Hafner, Jergesen HE, Wilson L, Lamoreux LW, Roberts R (1983) Evaluation of problems and needs of veteran lower-limb amputees in the San Francisco Bay Area during the period 1977-1980 . J Rehabil Res Dev 2(1): 57-71.
15. Miff SC, Hansen AH, Childress DS, Gard SA, Meier MR (2007) RollOver Shapes of the Able-Bodied Knee Ankle Foot System During Gait Initiation, Steady-State Walking, and Gait Termination. Gait Posture 27(2): 316-322.

\begin{tabular}{ll}
\hline BIOMEDICAL & Assets of Publishing with us \\
RESEARCHES & - Global archiving of articles \\
\hline
\end{tabular}

